\title{
Impact of Unbalance on Electrical and Torsional Resonances in Power Electronic Interfaced Wind Energy Systems
}

\author{
Zhixin Miao, Senior Member, IEEE
}

\begin{abstract}
Type 3 wind generators in series compensated networks could lead to subsynchronous resonances (SSR). Previous research focuses on balanced operation only. In this paper, impact of unbalance on Type 3 wind energy systems is investigated in two aspects: 1) impact on electric resonances and 2) impact on torsional resonances. In the first aspect, impedance models of the system in DQ domain and phase domain are developed. Particularly, negative sequence impedances are examined. Nyquist stability criterion is applied to detect possible resonances. In the second aspect, transfer functions of the electromagnetic torque versus the rotating speed due to negative sequence components are developed. The impact of negative sequence components on electromechanical damping is then analyzed. The analysis results are verified by time-domain simulation results.
\end{abstract}

Index Terms-Doubly fed induction generator, impedance model, subsynchronous resonance, torsional resonance, wind generation.

\section{INTRODUCTION}

$\mathbf{S}$ UBSYNCHRONOUS resonance (SSR) oscillations were observed in Type 3 wind farms by the industry recently. In [1], an event led to such phenomena was described and the recorded voltage and current waveforms with SSR oscillations are presented. A Type 3 wind farm is connected to a transmission path with two parallel lines. One line is equipped with series compensation (fixed capacitors). Due to a fault, the other line was tripped and the compensated line is directly connected to the wind farm. SSR oscillations started and kept increasing.

SSR phenomena in series compensated Type 3 wind energy systems have been studied in the authors' previous research [2]-[5]. In [2], DQ based system models in differential algebraic equations are developed. In [3], eigenvalue analysis is conducted to identify various system modes based on the DQ model. The finding from the research leads to the conclusion that SSR in series compensated Type 3 wind energy systems are mainly electrical resonances. Torsional interactions are rare due to the small values of the elastic coefficients of wind turbine shafts. The frequency of the torsional mode is low $(<3 \mathrm{~Hz})$. In

Manuscript received July 09, 2012; revised December 16, 2012; accepted January 14, 2013. Date of publication March 07, 2013; date of current version July 18, 2013. Paper no. TPWRS-00789-2012.

The author is with the Department of Electrical Engineering, University of South Florida, Tampa, FL 33620 USA (e-mail: zmiao@usf.edu).

Color versions of one or more of the figures in this paper are available online at $\mathrm{http}: / /$ ieeexplore.ieee.org.

Digital Object Identifier 10.1109/TPWRS.2013.2243174 order to have torsional interactions, the network has to have a very high compensation level to reach a high network frequency $(>47 \mathrm{~Hz}$ ). The finding from the previous research [2], [3] also indicates that rotor side converter (RSC) current control loops, wind speed and compensation level all have significant impact on SSR stability.

Since the SSR phenomenon is related mainly on the electrical systems, impedance based Nyquist stability analysis is carried out in [4] where the slip of an induction generator is expressed in Laplace domain based on intuition. In the most recent research [5], the positive-sequence impedance model for a Type 3 wind generator is derived rigorously from the DQ model. The finding is that the expression for the slip in [4] is accurate. Further, impacts of wind speed, compensation level and current controller parameters are studied using impedance based Nyquist criterion in [5]. Positive-sequence impedance based stability analysis carried out in [4] and [5] focuses on phenomena in balanced systems. Such study can answer the question whether there will be SSR stability (instability) when the system is under balanced three-phase operating conditions. However, the analysis cannot answer this question: will the system have SSR stability (instability) problems when subjected to unbalanced operation. To be able to answer such question, negative sequence impedance based analysis should be carried out. In addition, will negative sequence components increase or decrease electro-mechanical damping? Therefore, torque/speed transfer function based analysis will also be carried out.

The objective of this research is to investigate the impact on electrical and torsional resonances in series compensated Type 3 wind energy systems due to unbalanced operation. Impedance models in DQ domain and phase domain will be developed. Particularly, negative sequence impedance models will be derived. Nyquist stability criterion will then be applied to judge whether the system is SSR stable or not due to negative sequence components. In addition, impact of negative sequence components on torsional resonances is also examined using torque/speed transfer function.

The rest of the paper is organized as follows. Section II presents the impedance models in DQ and phase domains. Section III presents the torque/speed transfer function. Analysis of negative sequence component impact will be presented in these two sections. Section IV presents a time-domain simulation case study. An unbalanced operating condition will be initiated and the system dynamic performance will be compared with that under balance operating condition. Section $V$ presents the conclusion of this paper. 

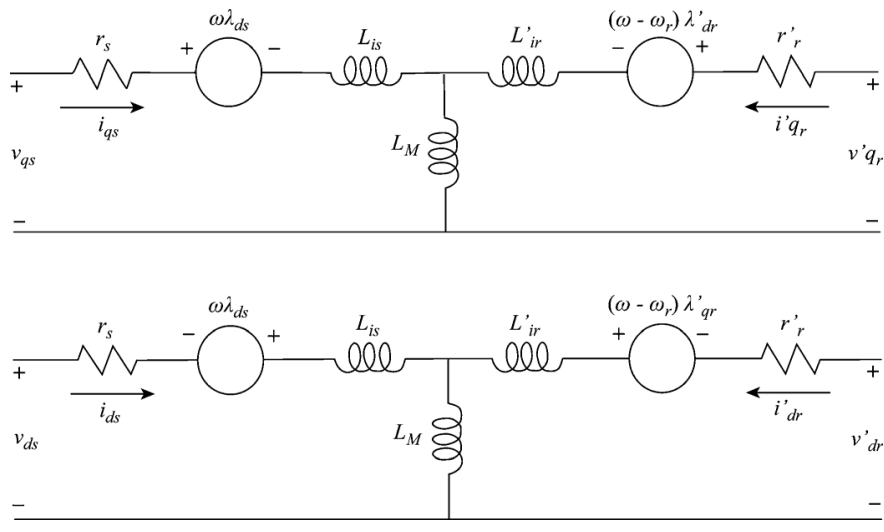

Fig. 1. The qd-axis induction machine circuits.

\section{IMPEDANCE MODEL}

Impedance model can be developed in both DQ domain and phase domain [6]-[8]. DQ domain based impedance model is more general and can handle unsymmetrical systems [6]. For an induction machine, due to the symmetry of its rotor and stator, its impedance models can also expressed in phase domain: positive sequence impedance and negative sequence impedance. In this section, these two models are derived and presented.

\section{A. DQ Domain}

This subsection is devoted to develop the impedance model of an induction machine based on the widely known DQ model presented in the classic textbook [9]. The qd-axis circuits are shown in Fig. 1.

The stator voltage, current and flux linkage relation can be expressed as

$$
\begin{aligned}
& v_{q s}=r_{s} i_{q s}+\omega \lambda_{d s}+p \lambda_{q s} \\
& v_{d s}=r_{s} i_{d s}-\omega \lambda_{q s}+p \lambda_{d s}
\end{aligned}
$$

where

$$
\begin{aligned}
& \lambda_{q s}=L_{l s} i_{q s}+M\left(i_{q s}+i_{q r}^{\prime}\right) \\
& \lambda_{d s}=L_{l s} i_{d s}+M\left(i_{d s}+i_{d r}^{\prime}\right) .
\end{aligned}
$$

The rotor voltage, current and flux linkage relation can be expressed as

$$
\begin{aligned}
& v_{q r}^{\prime}=r_{r}^{\prime} i_{q r}+\left(\omega-\omega_{m}\right) \lambda_{d r}^{\prime}+p \lambda_{q r} \\
& v_{d r}^{\prime}=r_{r}^{\prime} i_{d r}-\left(\omega-\omega_{m}\right) \lambda_{q r}^{\prime}+p \lambda_{d r}
\end{aligned}
$$

where

$$
\begin{aligned}
& \lambda_{q r}^{\prime}=L_{l r}^{\prime} i_{q r}^{\prime}+M\left(i_{q s}+i_{q r}^{\prime}\right) \\
& \lambda_{d r}^{\prime}=L_{l r}^{\prime} i_{d r}^{\prime}+M\left(i_{d s}+i_{d r}^{\prime}\right)
\end{aligned}
$$

Laplace transformation for (1) and (3) can be obtained:

$$
\begin{aligned}
& \mathbf{v}_{r}^{\prime}=C_{r 1} \mathbf{i}_{r}^{\prime}+C_{r 2} \mathbf{i}_{s} \\
& \mathbf{v}_{s}=C_{s 1} \mathbf{i}_{s}+C_{s 2} \mathbf{i}_{r}^{\prime}
\end{aligned}
$$

where $f_{r}^{\prime}=\left[f_{q r}^{\prime}, f_{d r}^{\prime}\right]^{T}, f_{s}=\left[f_{q s}, f_{d s}\right]^{T}$ and $f$ stands for the symbol of current or voltage:

$$
\begin{aligned}
C_{r 1} & =\left[\begin{array}{cc}
r_{r}^{\prime}+s L_{r r} & \left(\omega-\omega_{r}\right) L_{r r} \\
-\left(\omega-\omega_{r}\right) L_{r r} & r_{r}^{\prime}+s L_{r r}
\end{array}\right] \\
C_{r 2} & =\left[\begin{array}{cc}
s M & \left(\omega-\omega_{r}\right) M \\
-\left(\omega-\omega_{r}\right) M & s M
\end{array}\right] \\
C_{s 1} & =\left[\begin{array}{cc}
r_{s}+s L_{s s} & \omega L_{s s} \\
-\omega L_{s s} & r_{s}+s L_{s s}
\end{array}\right] \\
C_{s 2} & =\left[\begin{array}{cc}
s M & \omega M \\
-\omega M & s M
\end{array}\right] .
\end{aligned}
$$

In addition, the current control of the RSC can be expressed as

$$
\mathbf{v}_{r}^{\prime}=H(s)\left(\mathbf{i}_{r}^{*}-\mathbf{i}_{r}^{\prime}\right)
$$

From (5), the RSC current $\mathbf{i}_{r}^{\prime}$ can be expressed by the RSC voltage $\mathbf{v}_{r}^{\prime}$ and the stator current $\mathbf{i}_{s}$ :

$$
\mathbf{i}_{r}^{\prime}=C_{r 1}^{-1} \mathbf{v}_{r}^{\prime}-C_{r 1}^{-1} C_{r 2} \mathbf{i}_{s}
$$

Therefore, the stator voltage can be expressed by the stator current and the RSC voltage solely:

$$
\mathbf{v}_{s}=C_{s 2} C_{r 1}^{-1} \mathbf{v}_{r}^{\prime}+\left(C_{s 1}-C_{s 2} C_{r 1}^{-1} C_{r 2}\right) \mathbf{i}_{s} .
$$

Substitute $\mathbf{i}_{r}^{\prime}$ with the expression in (7), (6) becomes

$$
\mathbf{v}_{r}^{\prime}=\left(I+H C_{r 1}^{-1}\right)^{-1} H\left(\mathbf{i}_{r}^{*}+C_{r 1}^{-1} C_{r 2} \mathbf{i}_{s}\right) .
$$

Hence the stator voltage can be expressed in terms of the stator current and rotor current reference solely:

$$
\begin{aligned}
& \mathbf{v}_{s}=C_{s 2} C_{r 1}^{-1}\left(I+H C_{r 1}^{-1}\right)^{-1} H \mathbf{i}_{r}^{*} \\
& +\underbrace{\left[C_{s 2} C_{r 1}^{-1}\left(I+H C_{r 1}^{-1}\right)^{-1} H C_{r 1}^{-1} C_{r 2}+C_{s 1}-C_{s 2} C_{r 1}^{-1} C_{r 2}\right]}_{Z_{d f i g}} \mathbf{i}_{s} .
\end{aligned}
$$

The DQ impedance model for a three-phase RLC circuit can be expressed as

$$
Z_{\text {line }}=\left[\begin{array}{cc}
R+s L+\frac{s}{\left(s^{2}+\omega^{2}\right) C} & \omega L-\frac{\omega}{\left(s^{2}+\omega^{2}\right) C} \\
-\omega L+\frac{\omega}{\left(s^{2}+\omega^{2}\right) C} & R+s L+\frac{s}{\left(s^{2}+\omega^{2}\right) C}
\end{array}\right] .
$$

The Nyquist maps of the eigenvalues of $Z_{d f i g}^{-1} Z_{\text {line }}$ can be used to detect resonances and instability.

\section{B. Phase Domain}

In this subsection, positive and negative sequence impedance models of Type 3 wind generator and the network will be developed. For a three-phase system, the impedance model can be expressed in space vector or $q d$ reference frame. From the terminal of a network, when positive voltage is applied to the terminal, the observed impedance is positive sequence impedance. When negative sequence voltage is applied to the terminal, the observed impedance is negative sequence impedance.

When the system has both positive and negative sequence components, and let $\bar{V}_{p}$ and $\bar{V}_{n}$ be the positive and negative 
sequence components in $\bar{V}_{a}$ where $\bar{V}_{a}$ is the dynamic phasor of the phase a voltage at the fundamental frequency.

The phasors are related to the instantaneous variables as follows:

$$
\left\{\begin{array}{l}
v_{a}(t)=\left(\bar{V}_{p}+\bar{V}_{n}\right) e^{j \omega t}+\left(\bar{V}_{p}^{*}+\bar{V}_{n}^{*}\right) e^{-j \omega t} \\
v_{b}(t)=\left(a^{2} \bar{V}_{p}+a \bar{V}_{n}\right) e^{j \omega t}+\left(a \bar{V}_{p}^{*}+a^{2} \bar{V}_{n}^{*}\right) e^{-j \omega t} \\
v_{c}(t)=\left(a \bar{V}_{p}+a^{2} \bar{V}_{n}\right) e^{j \omega t}+\left(a^{2} \bar{V}_{p}^{*}+a \bar{V}_{n}^{*}\right) e^{-j \omega t}
\end{array}\right.
$$

where $a=e^{j 2 \pi / 3}$.

The space vector of the terminal voltage is defined as

$$
\vec{V}(t)=\left(\frac{2}{3}\right)\left(v_{a}(t)+a v_{b}(t)+a^{2} v_{c}(t)\right) .
$$

The space vector can also be expressed in terms of positive and negative sequence phasors:

$$
\vec{V}(t)=2 \bar{V}_{p} e^{j \omega t}+2 \bar{V}_{n}^{*} e^{-j \omega t} .
$$

In Laplace domain, the voltage and current space vectors can be written as

$$
\left\{\begin{array}{l}
\vec{V}(s)=2 \bar{V}_{p}(s-j \omega)+2 \bar{V}_{n}^{*}(s+j \omega) \\
\vec{I}(s)=2 \bar{I}_{p}(s-j \omega)+2 \bar{I}_{n}^{*}(s+j \omega) .
\end{array}\right.
$$

When there is only positive sequence or negative sequence, then the impedance observed based on space vectors should have the following relationship with the impedance observed based on the complex vectors or dynamic phasor:

$$
\left\{\begin{array}{l}
\text { Positive }: Z_{p}=\frac{\vec{V}(s)}{\vec{I}(s)}=\frac{\bar{V}_{p}(s-j \omega)}{\bar{I}_{p}(s-j \omega)} \\
\text { Negative }: Z_{n}=\frac{\vec{V}(s)}{\vec{I}(s)}=\frac{\bar{V}_{n}^{*}(s+j \omega)}{\bar{I}_{n}^{*}(s+j \omega)} .
\end{array}\right.
$$

Since the expression of $V_{p} / I_{p}$ and $V_{n} / I_{n}$ are the same, it can be found that

$$
Z_{p}=Z_{n}^{*}
$$

1) RLC Circuit Impedance Model: For a three-phase RLC circuit, the impedance model observed in space vector can be expressed as

$$
Z_{\text {line }, p}=Z_{\text {line }, n}=R+s L+\frac{1}{s C} .
$$

The above expression also fits (15). Since there is no imaginary part for the positive sequence expression, the negative sequence and the positive sequence expressions are the same.

2) Induction Machine Impedance Model: Two complex vectors $\bar{V}_{q d s}=v_{q}-j v_{d}$ and $\bar{I}_{q d s}=i_{q}-j i_{d}$ are defined. Using the complex vector expression, and transforming (3) into Laplace domain leads to

$\bar{V}_{r}^{\prime}(s)=\left(r_{r}^{\prime}+\left(s+j\left(\omega-\omega_{r}\right)\right) L_{r r}\right) \bar{I}_{r}^{\prime}(s)+M\left(s+j\left(\omega-\omega_{r}\right)\right) \bar{I}_{s}(s)$

where $L_{s s}=L_{l s}+M$ and $L_{r r}^{\prime}=L_{l r}^{\prime}+M$. Hence $I_{r}^{\prime}(s)$ can be expressed by $\bar{V}_{r}^{\prime}$ and $\bar{I}_{s}$.
Converting (1) into Laplace domain and substitute the static current space vector according to (17) leads to

$$
\begin{gathered}
\bar{V}_{s}=\underbrace{\left[r_{s}+(s+j \omega)\left(L_{s s}-\frac{M^{2}\left[s+j\left(\omega-\omega_{r}\right)\right]}{r_{r}^{\prime}+\left[s+j\left(\omega-\omega_{r}\right) L_{r r}\right]}\right)\right]}_{Z} \bar{I}_{s} \\
+\frac{(s+j \omega) M}{r_{r}^{\prime}+\left[s+j\left(\omega-\omega_{r}\right)\right] L_{r r}} \bar{V}_{r}^{\prime} .
\end{gathered}
$$

Therefore, a Thevenin equivalent circuit for the induction machine can be developed. Notice that the impedance $Z(s)$ is based on DQ0 reference frame. Since the RLC circuit is expressed in abc reference frame, it will be convenient that there is also an impedance model based on abc reference frame.

In space vector the stator voltage can be expressed as follows by replacing $s$ by $s-j \omega$ for positive sequence and replacing $s$ by $s+j \omega$ for negative sequence. Positive sequence:

$$
\begin{aligned}
\overrightarrow{V(s)}= & \bar{V}_{s}(s-j \omega) \\
= & \underbrace{\left[r_{s}+s\left(L_{s s}-\frac{\left.M^{2}\left[s-j \omega_{r}\right)\right]}{\left.r_{r}^{\prime}+\left[s-j \omega_{r}\right) L_{r r}\right]}\right)\right]}_{Z} \vec{I}_{s} \\
& +\frac{s M}{r_{r}^{\prime}+\left(s-j \omega_{r}\right) L_{r r}} \vec{V}_{r}^{\prime} .
\end{aligned}
$$

Therefore, the positive sequence impedance model is

$$
\begin{aligned}
Z_{d f i g, p} & =r_{s}+s\left(L_{l s}+M-\frac{M^{2}}{\frac{r_{r}^{\prime}}{s-j \omega_{r}}+L_{l r}^{\prime}+M}\right) \\
& =r_{s}+s L_{l s}+s M \frac{\frac{r_{r}^{\prime}}{s-j \omega_{r}}+L_{l r}^{\prime}}{\frac{r_{r}^{\prime}}{s-j \omega_{r}}+L_{l r}^{\prime}+M} .
\end{aligned}
$$

The Negative sequence impedance model is

$$
\begin{aligned}
Z_{d f i g, n} & =r_{s}+s\left(L_{l s}+M-\frac{M^{2}}{\frac{r_{r}^{\prime}}{s+j \omega_{r}}+L_{l r}^{\prime}+M}\right) \\
& =r_{s}+s L_{l s}+s M \frac{\frac{r_{r}^{\prime}}{s+j \omega_{r}}+L_{l r}^{\prime}}{\frac{r_{r}^{\prime}}{s+j \omega_{r}}+L_{l r}^{\prime}+M} .
\end{aligned}
$$

3) Converter Impedance Model: Cascaded control loops are used in converters in wind generators. The control loops consist of the fast inner current control loops and the slow outer power/voltage control loops. The current control loops usually have bandwidths at or greater than $100 \mathrm{~Hz}$ while the outer control loops usually have bandwidths less than several Hz. For SSR studies, the study dynamics is considered faster than the outer control loops. Thus, the outer control is considered to be constant and will not be included in the impedance model.

For the vector current control scheme in Fig. 2, the $q d$-axis voltage and current relationship is

$$
\begin{aligned}
& v_{q}=\left(i_{q}^{*}-i_{q}\right) H_{i}(s)-K_{d} i_{d} \\
& v_{d}=\left(i_{d}^{*}-i_{d}\right) H_{i}(s)+K_{d} i_{q} .
\end{aligned}
$$

This leads to the expression in complex vector:

$$
\bar{V}_{q d}=\bar{I}_{q d}^{*} H_{i}(s)-\left(H_{i}(s)-j K_{d}\right) \bar{I}_{q d} .
$$




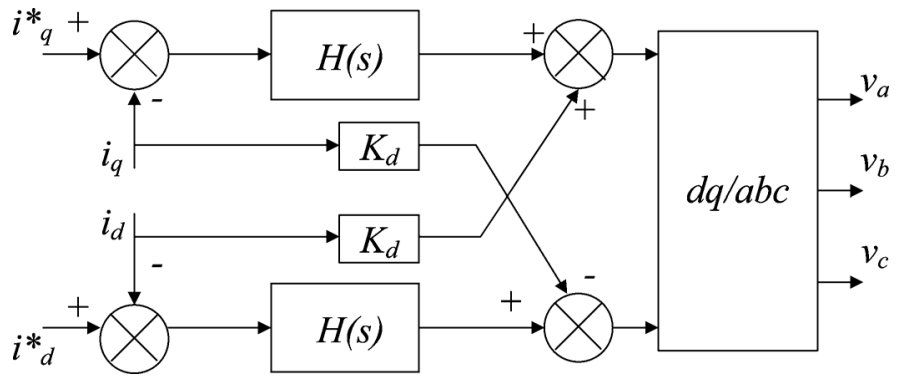

Fig. 2. Converter current loop control.

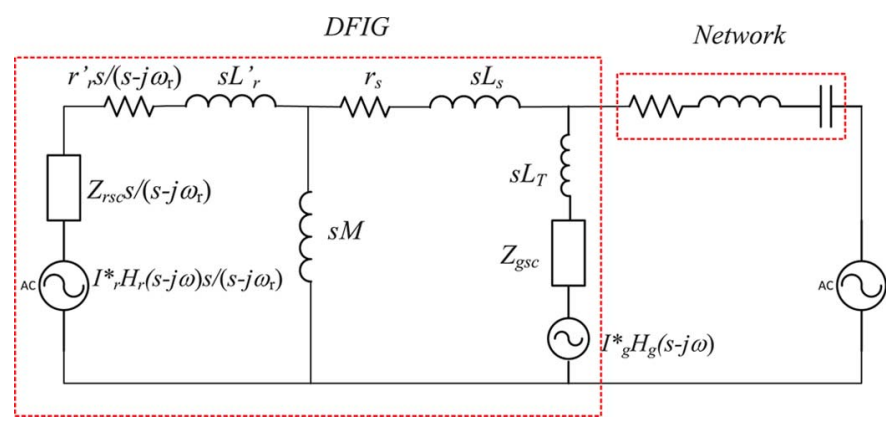

Fig. 3. Overall circuit model for positive sequence.

To view the current controlled converter from space vector, then

$$
\left\{\begin{array}{l}
\text { Positive }: \vec{V}=\vec{I}^{*} H_{i}(s-j \omega)-\left(H_{i}(s-j \omega)-j K_{d}\right) \vec{I} \\
\text { Negative }: \vec{V}=\vec{I}^{*} H_{i}(s+j \omega)-\left(H_{i}(s+j \omega)+j K_{d}\right) \vec{I}
\end{array}\right.
$$

For the GSC converter at positive sequence scenarios, it can be represented by a voltage source $\vec{I}_{g}^{*} H_{g}(s-j \omega)$ behind an impedance $Z_{g s c}$ where $Z_{g s c}=H_{g}(s-j \omega)-j K_{d g}$. At negative sequence scenarios, the GSC converter can be represented by a voltage source $\vec{I}_{g}^{*} H_{g}(s+j \omega)$ behind an impedance $Z_{g s c}$ where $Z_{g s c}=H_{g}(s+j \omega)+j K_{d g}$.

For the RSC converter at positive sequence scenarios, it can also be represented by a voltage source $\vec{I}_{r}^{*} H_{r}(s-j \omega)$ behind an impedance $Z_{r s c}$ where $Z_{r s c}=H_{r}(s-j \omega)-j K_{d r}$. The negative sequence impedance of the RSC is the conjugate of $Z_{r s c}$ and can be expressed as $H_{r}(s+j \omega)+j K_{d r}$.

\section{Overall Circuit}

The overall circuit for positive sequence is now shown in Fig. 3.

The overall circuit for negative sequence is now shown in Fig. 4.

For the study system in Fig. 5 [2], the bode plots of the negative sequence impedances are shown in Fig. 6. Two network impedances are presented: one is at $25 \%$ compensation level while the other is at $70 \%$ compensation level. Two DFIG impedances are presented: one is at $75 \%$ nominal speed while the other is at $95 \%$ nominal speed. From the Bode plots, the two Bode plots almost match each other. Therefore wind speed has negligible impact on the DFIG impedance. The network impedance magnitudes and the DFIG impedance magnitudes meet at $150 \mathrm{rad} / \mathrm{s}$ ( $24 \mathrm{~Hz}, 25 \%$ compensation level) and $250 \mathrm{rad} / \mathrm{s}(40 \mathrm{~Hz}, 75 \%$ compensation level). There are phase margin for $Z_{\text {line }} / Z_{\text {dfig }}$.

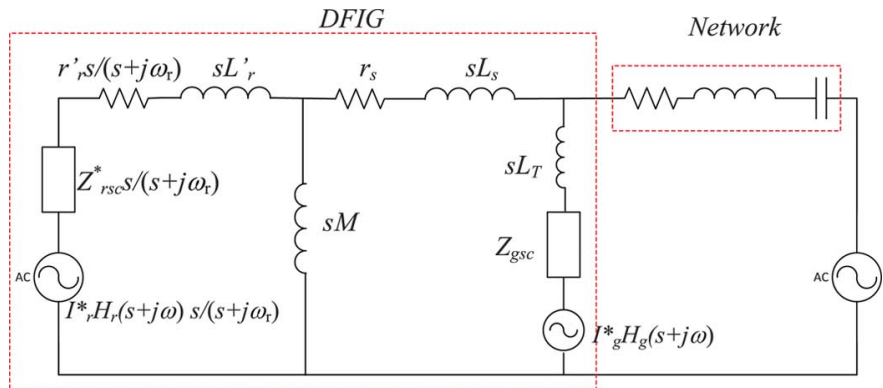

Fig. 4. Overall circuit model for negative sequence.

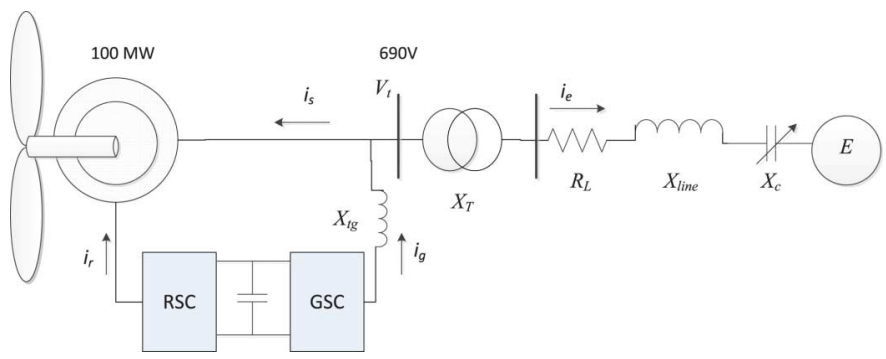

Fig. 5. Study system.

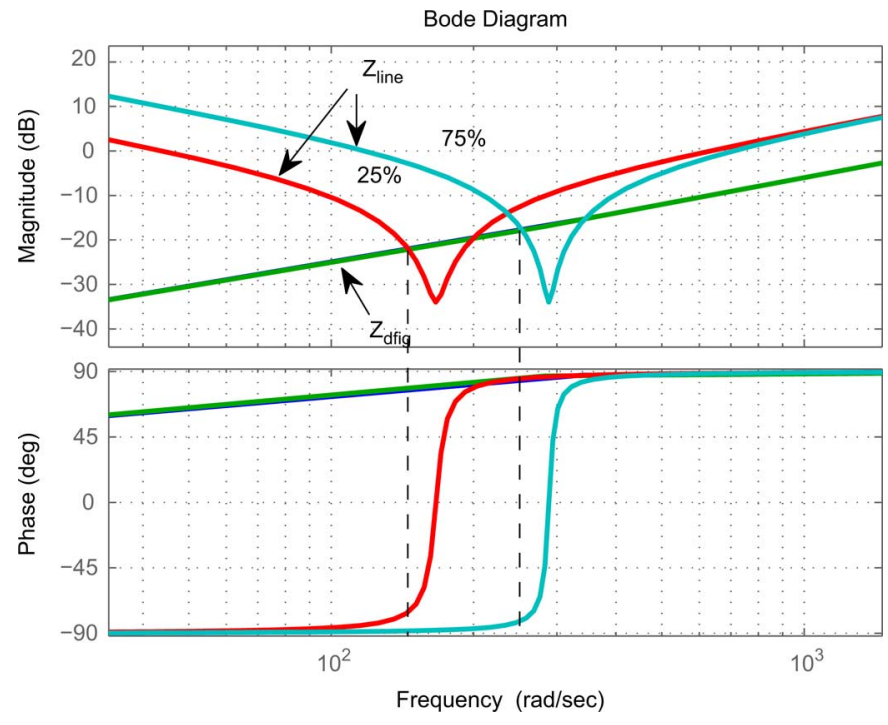

Fig. 6. Bode plots of negative sequence impedances.

Hence the negative sequence impedance will not cause SSR instability.

A comparison of the positive and negative sequence impedances of the DFIG is shown in Fig. 7. From this figure, it is obvious that the positive sequence impedance of the DFIG could be greater than $90^{\circ}$ at the gain cross frequencies. This indicates that it is the positive sequence impedance that could cause SSR instability.

The DQ domain impedances are 2-by-2 matrices. The Nyquist plot of the eigenvalue of $Z_{\text {dfig }}^{-1} Z_{\text {line }}$ is shown in Fig. 8. The unit circle is also plotted. It is detected that a $36-\mathrm{Hz}$ resonance may appear in DQ-domain. This resonance will appear as $24-\mathrm{Hz}$ positive-sequence resonance in abc domain. Thus, the DQ-domain impedance model based analysis reaches the same 


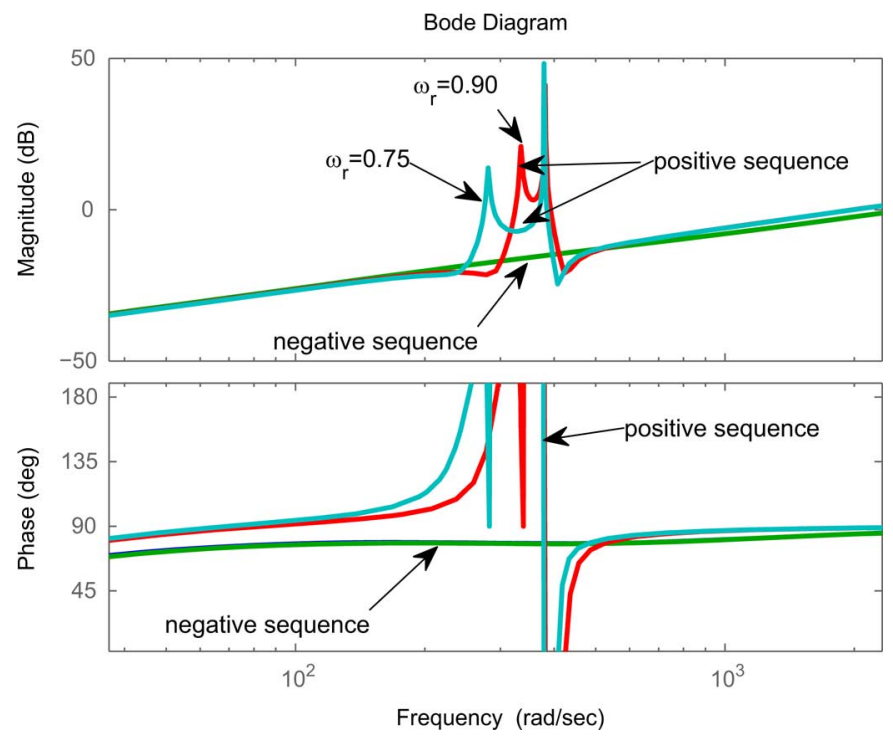

Fig. 7. Bode plots of positive and negative sequence impedances.

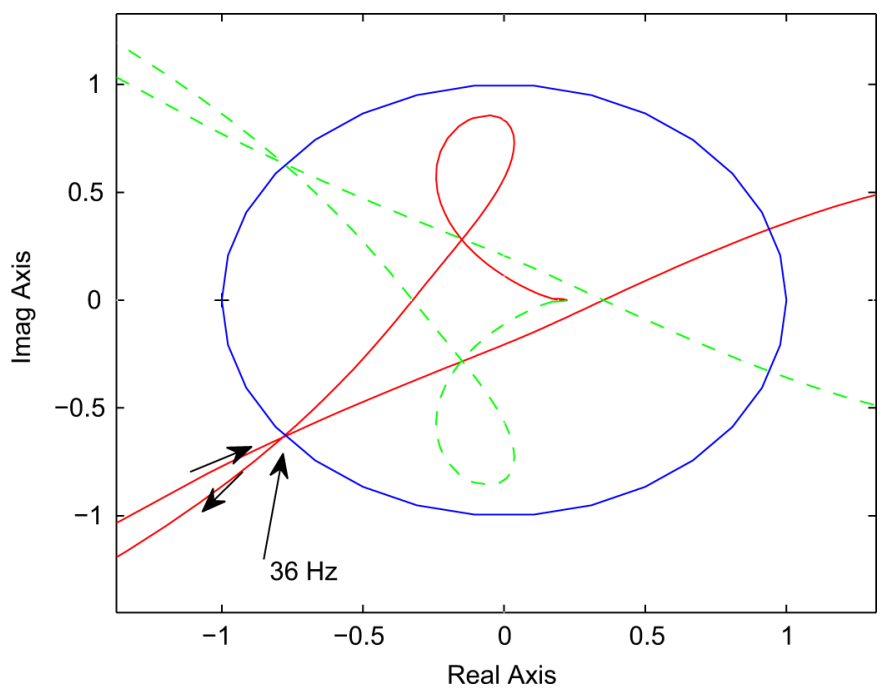

Fig. 8. Bode plots of an eigenvalue of $Z_{\text {df } i g}^{-1} Z_{\text {line }}$ in DQ-domain. Compensation level $25 \%$, rotating speed $0.90 \mathrm{pu}$.

conclusion from the positive and negative sequence impedance based analysis.

\section{TORQUe SPEED RELATIONSHIP}

To investigate the impact of negative sequence components on torsional interaction, transfer function of the electromagnetic torque $\left(T_{e}\right)$ versus the rotating speed $\left(\omega_{r}\right)$ is developed in this section. The interactions of torque and speed relationship is represented in Fig. 9 [10], where $G_{e}(s)$ represents the relationship of $T_{e}$ versus $\omega_{r}$ through electrical system and $G_{m}(s)$ represents the relationship of $\omega_{r}$ and $T_{e}$ through mechanical system. Note the positive feedback is due to the motor convention used in this paper. When operated as a generator, $T_{e}$ is a negative number.

Therefore the electromagnetic torque and speed transfer function is

$$
G_{c l}(s)=\frac{G_{e}(s)}{1-G_{m}(s) G_{e}(s)} .
$$

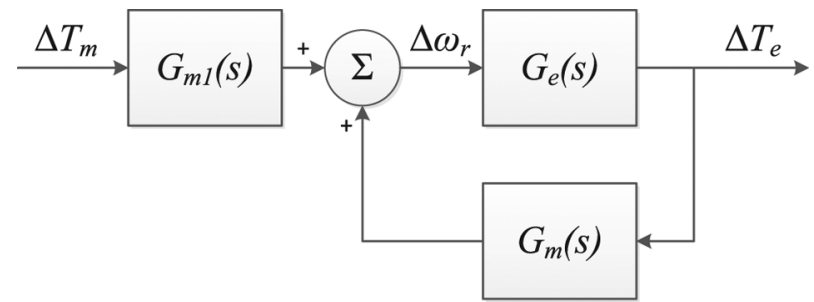

Fig. 9. Torque and rotating speed relationship.

\section{A. Mechanical System}

A two-mass system [11], [12] is used to the represent torsional dynamics given by

$$
\begin{array}{r}
{\left[\begin{array}{c}
\Delta \dot{\omega}_{t} \\
\Delta \dot{\omega}_{r} \\
T_{12}
\end{array}\right]=\left[\begin{array}{ccc}
\frac{-D_{t}-D_{t g}}{2 H_{t}} & \frac{D_{t g}}{2 H_{t}} & \frac{-1}{2 H_{t}} \\
\frac{D_{t g}}{2 H_{g}} & \frac{-D_{g}-D_{t g}}{2 H_{g}} & \frac{1}{2 H_{g}} \\
K_{t g} \omega_{e} & -K_{t g} \omega_{e} & 0
\end{array}\right]\left[\begin{array}{c}
\Delta \omega_{t} \\
\Delta \omega_{r} \\
T_{12}
\end{array}\right]} \\
+\left[\begin{array}{c}
\frac{T_{m}}{2 H_{t}} \\
\frac{-T_{e}}{2 H_{g}} \\
0
\end{array}\right]
\end{array}
$$

where $\omega_{t}$ and $\omega_{r}$ are the turbine and generator rotor speed, respectively; $P_{m}$ and $P_{e}$ are the mechanical power of the turbine and the electrical power of the generator, respectively; $T_{12}$ is an internal torque of the model; $H_{t}$ and $H_{g}$ are the inertia constants of the turbine and the generator, respectively; $D_{t}$ and $D_{g}$ are the mechanical damping coefficients of the turbine and the generator, respectively; $D_{t g}$ is the damping coefficient of the flexible coupling (shaft) between the two masses; $K_{t g}$ is the shaft stiffness. From (27), the transfer functions $G_{m 1}=\Delta T_{m} / \Delta \omega_{r}$ and $G_{m}=\Delta T_{e} / \Delta \omega_{r}$ can be found.

\section{B. Electrical System}

Torque-speed transfer function has been used in [13] to examine torsional interactions. The voltage and current equations in (1) and (3) for stator and rotor in DQ domain are linearized. The resulting linearized differential equations are as follows:

$$
\frac{d}{d t} \Delta \mathbf{i}_{s r}=A_{1} \Delta \mathbf{i}_{s r}+A_{2} \Delta \omega_{r}+B_{1} \Delta \mathbf{v}_{s}+B_{2} \Delta \mathbf{v}_{r}^{\prime}
$$

where $\mathbf{i}_{s} r=\left[i_{q s}, i_{d s}, i_{q r}, i_{d r}\right]^{T}, \mathbf{v}_{s}=\left[v_{q s}, v_{d s}\right]^{T}$ and $\mathbf{v}_{r}^{\prime}=$ $\left[v_{q r}^{\prime}, v_{d r}^{\prime}\right]^{T}$.. In Laplace domain, the above equation becomes

$$
\left(s I-A_{1}\right) \Delta \mathbf{i}_{s r}=A_{2} \Delta \omega_{r}+B_{1} \Delta \mathbf{v}_{s}+B_{2} \Delta \mathbf{v}_{r}^{\prime} .
$$

Consider the current control loops in the RSC, the RSC voltage can be expressed as

$$
\Delta \mathbf{v}_{r}^{\prime}=H_{r s c}(s) \Delta \mathbf{i}_{r}^{*}-Z_{r s c} \Delta \mathbf{i}_{r} .
$$

If we assume that the converter outer control loops can be ignored, then the reference signal $\mathbf{i}_{r}^{*}$ are constants and its deviation is zero.

Substituting $\Delta \mathbf{v}_{r}^{\prime}$ in (28) by (29) leads to

$$
\begin{aligned}
\Delta \mathbf{i}_{s r}= & \left(s I-A_{1}+B_{2}\left[\begin{array}{ll}
\mathbf{0} & Z_{r s c}
\end{array}\right]\right)^{-1} \\
& \times\left(A_{2} \Delta \omega_{r}+B_{1} \Delta v_{s}\right) \\
= & G_{v i} \Delta V_{s}+G_{\omega i} \Delta \omega_{r} .
\end{aligned}
$$


It is obvious to find the expression of $\Delta \mathbf{i}_{s}$ in terms of the rotating speed and the stator voltage:

$$
\Delta \mathbf{i}_{s}=G_{v i s} \Delta \mathbf{v}_{s}+G_{\omega i s} \Delta \omega_{r}
$$

The expressions can be developed for both positive and negative sequences. For positive sequence, the DQ reference frame $\left(d q^{+}\right)$is rotating counter clockwise at nominal frequency $\omega$. While for negative sequence, the DQ reference frame is rotating clockwise at the nominal frequency $\omega$. The major differences in expressions are $A_{1}$ and $Z_{r s c}$. For positive sequence, since the vector control is based on the same reference frame, $Z_{r s c}^{+}=H(s)=K_{p}+K_{i} / s$. However, for negative sequence, since the vector control is based on $d q^{+}$while the model is based on $d q^{-}$, the impedance of the RSC should be converted to $q d^{-}$. Hence $Z_{r s c}^{-}=H(s-j 2 \omega)=K_{p}+K_{i} /(s-j 2 \omega)$ :

$$
\left\{\begin{array}{l}
\Delta i_{s, p}^{+}=G_{v i s}^{+} \Delta v_{s, p}+G_{\omega i s, p}^{+} \Delta \omega_{r} \\
\Delta i_{s, n}^{-}=G_{v i s}^{-} \Delta v_{s, n}+G_{\omega i s, n}^{-} \Delta \omega_{r} .
\end{array}\right.
$$

The electromagnetic torque of an induction machine can be expressed by the stator and the rotor currents:

$$
T_{e}=\frac{X_{M}}{2}\left(i_{q s} i_{d r}-i_{d s} i_{q r}\right) .
$$

When there is only positive sequence, $T_{e}$ is a dc variable. When there is only negative sequence, $T_{e}$ is also a dc variable. To evaluate the impact of positive and negative sequence components on the dc component of $T_{e}$, the following definitions are made:

$$
\left\{\begin{array}{l}
T_{e, d c 1}=\frac{X_{M}}{2}\left(i_{q s, p}^{+} i_{d r, p}^{+}-i_{d s, p}^{+} i_{q r, p}^{+}\right) \\
T_{e, d c 2}=\frac{X_{M}}{2}\left(i_{q s, n}^{-} i_{d r, n}^{-}-i_{d s, n}^{-} i_{q r, n}^{-}\right) .
\end{array}\right.
$$

The linearized expressions are as follows:

$$
\begin{aligned}
\Delta T_{e, d c 1} & =\frac{X_{M}}{2}\left[i_{d r, p}^{+},-i_{q r, p}^{+},-i_{d s, p}^{+}, i_{q s, p}^{+}\right] \Delta \mathbf{i}_{s r, p}^{+} \\
& =G_{v t}^{+} \Delta v_{s, p}^{+}+G_{\omega t}^{+} \Delta \omega_{r} \\
\Delta T_{e, d c 2} & =\frac{X_{M}}{2}\left[i_{d r, n}^{-},-i_{q r, n}^{-},-i_{d s, n}^{-}, i_{q s, n}^{-}\right] \Delta \mathbf{i}_{s r, n}^{-} \\
& =G_{v t}^{-} \Delta v_{s, n}^{-}+G_{\omega t}^{-} \Delta \omega_{r} .
\end{aligned}
$$

In addition, the stator voltage can be expressed by the stator current through the network equation if the current through the GSC is ignored:

$$
\left\{\begin{array}{l}
\Delta v_{s, p}^{+}=-Z_{\text {line }}^{+} \Delta i_{s, p}^{+} \\
\Delta v_{s, n}^{-}=-Z_{\text {line }}^{-} \Delta i_{s, n}^{-}
\end{array}\right.
$$

where

$$
\left\{\begin{array}{l}
Z_{\text {line }}^{+}=\left[\begin{array}{cc}
R+s L+\frac{s}{\left(s^{2}+\omega^{2}\right) C} & \omega L-\frac{\omega}{\left(s^{2}+\omega^{2}\right) C} \\
-\omega L+\frac{\omega}{\left(s^{2}+\omega^{2}\right) C} & R+s L+\frac{s}{\left(s^{2}+\omega^{2}\right) C}
\end{array}\right] \\
Z_{\text {line }}^{-}=\left[\begin{array}{cc}
R+s L+\frac{\omega}{\left(s^{2}+\omega^{2}\right) C} & -\omega L+\frac{\omega}{\left(s^{2}+\omega^{2}\right) C} \\
\omega L-\frac{\omega}{\left(s^{2}+\omega^{2}\right) C} & R+s L+\frac{s}{\left(s^{2}+\omega^{2}\right) C}
\end{array}\right] .
\end{array}\right.
$$

According to (36), (32) and (37), the torque versus the speed transfer functions $G_{e}(s)$ can be found:

$$
G_{e}^{+}(s)=-G_{v t}^{+}\left(I+Z_{\text {line }}^{+} G_{v i s}^{+}\right)^{-1} Z_{\text {line }}^{+} G_{w i s}^{+}+G_{w t}^{+}
$$

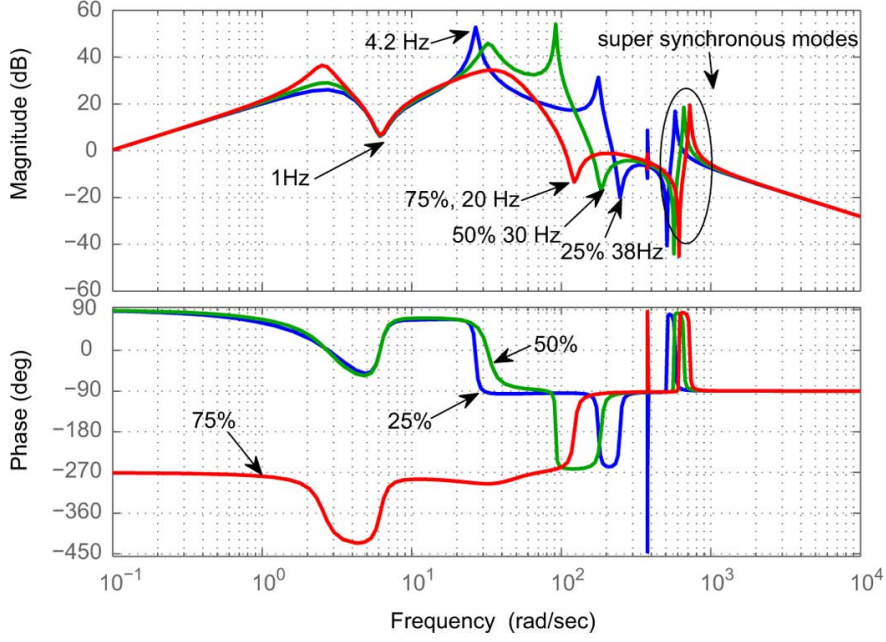

Fig. 10. Bode plots of torque/speed relationship considering positive sequence components only. Wind speed $9 \mathrm{~m} / \mathrm{s}$.

$$
G_{e}^{-}(s)=-G_{v t}^{-}\left(I+Z_{\text {line }}^{-} G_{v i s}^{-}\right)^{-1} Z_{\text {line }}^{-} G_{w i s}^{-}+G_{w t}^{-} .
$$

\section{Frequency Domain Analysis}

Bode plots of the torque/speed transfer function $G_{c l}(s)$ will be plotted. The operating conditions are: wind speed $9 \mathrm{~m} / \mathrm{s}$, compensation level $(25 \%, 50 \%$, and $75 \%)$. Two scenarios will be examined: with positive sequence components only and with negative sequence components only. In the first case, $G_{c l}(s)=$ $G_{e}^{+}(s) / 1-G_{m}(s) G_{e}^{+}(s)$ and in the second case, $G_{c l}(s)=$ $G_{e}^{-}(s) / 1-G_{m}(s) G_{e}^{-}(s)$.

Fig. 10 presents Bode plots of torque/speed relationship considering positive sequence components only. Three scenarios with different compensation levels are presented. The Bode plots indicate that the system has several oscillation modes, including a mode related to mechanical dynamics with less than $1 \mathrm{~Hz}$, a mode related to the electromechanical interaction at 4-6 Hz, the subsynchronous resonance (SSR) mode $(\sim 38 \mathrm{~Hz}$ at $25 \%, \sim 30 \mathrm{~Hz}$ at $50 \%$ and $\sim 20 \mathrm{~Hz}$ at $75 \%$ ) and the supersynchronous mode. Note that the frequency of the SSR mode in torque has the complementary frequency of the SSR frequency in voltages and currents. For example, the SSR frequency of the electric system at $9 \mathrm{~m} / \mathrm{s}$ at $25 \%$ compensation level is 24 Hz. The resonance frequency can be found in Fig. 6 the phase domain impedance Bode plot. In the DQ domain impedance Bode plots in Fig. 8, this resonance frequency becomes $36 \mathrm{~Hz}$. In Fig. 10, the SSR frequency of $9 \mathrm{~m} / \mathrm{s} 25 \%$ case is also around $36 \mathrm{~Hz}$.

Fig. 11 presents Bode plots of torque/speed relationship considering negative sequence components only. These Bode plots are obtained based on the linearized model where an initial negative sequence operating condition is assumed. The Bode plots in Fig. 10 are also shown here in dotted lines as comparison. It can be seen that magnitude wise, the negative sequence component's effect in the range of $1 \tilde{6} 0 \mathrm{~Hz}$ is insignificant compared to the positive sequence component. 


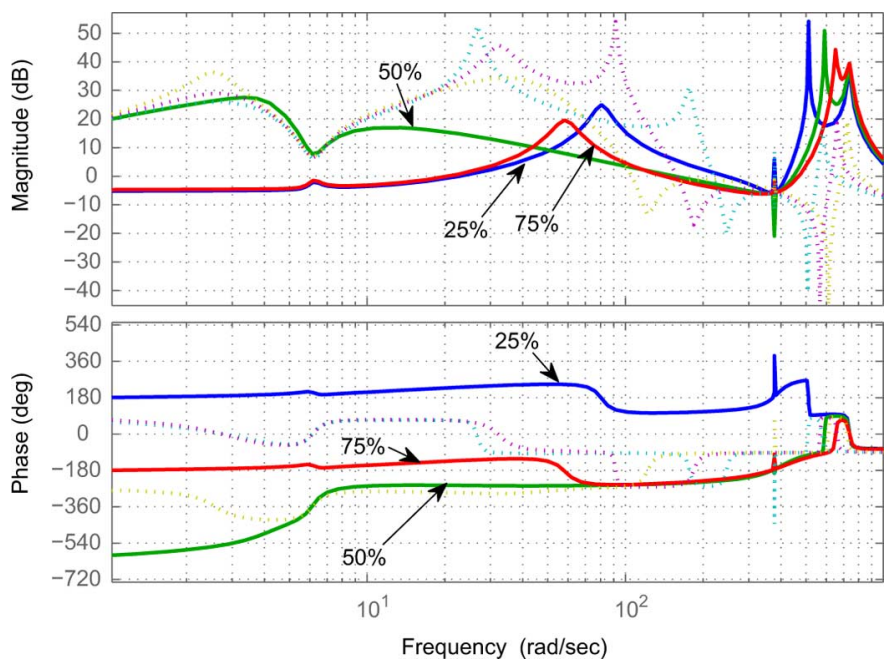

Fig. 11. Bode plots of torque/speed relationship considering only negative sequence components. Wind speed $9 \mathrm{~m} / \mathrm{s}$. Dotted lines correspond to positive sequence components.

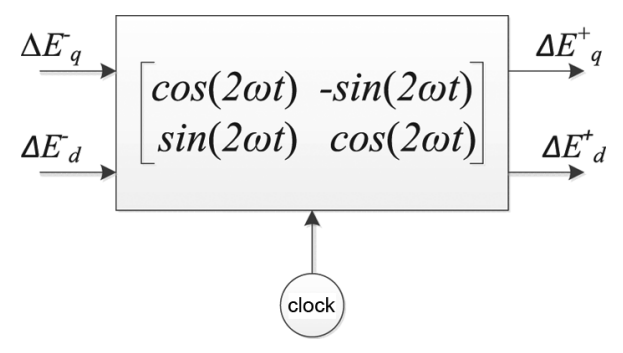

Fig. 12. $d q^{-}$to $d q^{+}$.

\section{Simulation Results}

In this section, time-domain simulation is carried out in Matlab/Simulink using nonlinear $d q$ based models developed in [2]. Two types of disturbances are applied to the test system where a Type 3 wind farm interconnected to a series compensated transmission line). The parameters of the test system can be found in [2, Appendix].

The wind speed is chosen to be $9 \mathrm{~m} / \mathrm{s}$ and the system compensation level is $25 \%$. Three cases are studied.

- In Case 1 , at $t=2 \mathrm{~s}$, a positive sequence voltage drop $(0.1$ $\mathrm{pu})$ is applied at the system voltage.

- In Case 2, at $t=2 \mathrm{~s}$, a negative sequence disturbance ( 0.1 $\mathrm{pu}$ ) is applied to the system voltage.

- In Case 3 , at $t=2 \mathrm{~s}$, a negative sequence disturbance $(0.5$ $\mathrm{pu}$ ) is applied to the system voltage.

The simulation model is built in $\mathrm{dq}$ synchronous reference frame. In Case 1, a step response is applied at the q-axis system voltage. While in Case 2 and Case 3, a step response is applied at the q-axis of system voltage of the $d q^{-}$reference frame. It is then transformed into $d q^{+}$reference frame as shown in Fig. 12.

The system dynamic responses will be presented. The simulation results are presented in the following figures. Fig. 13 presents the machine rotating speed dynamic responses. It can be observed that there are SSR mode at $36 \mathrm{~Hz}$ and a $4.5 \mathrm{-Hz}$ oscillation in the balanced disturbance case. When unbalanced disturbance is applied, the $120-\mathrm{Hz}$ oscillation is sustained as

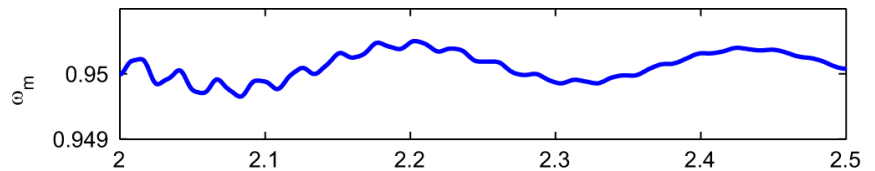

(a)

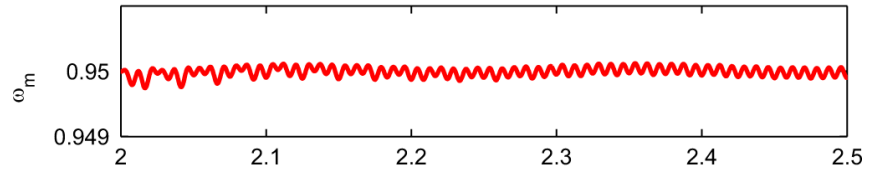

(b)

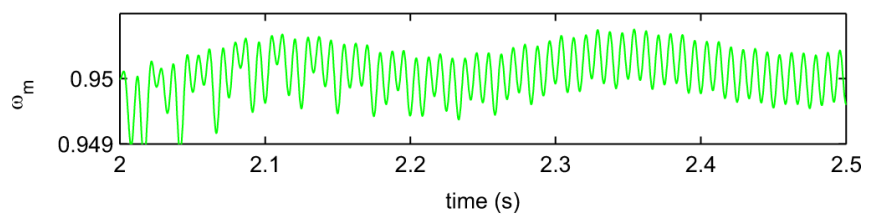

(c)

Fig. 13. Rotating speed when subject to a system voltage disturbance. a) Case 1. b) Case 2. c) Case 3 .
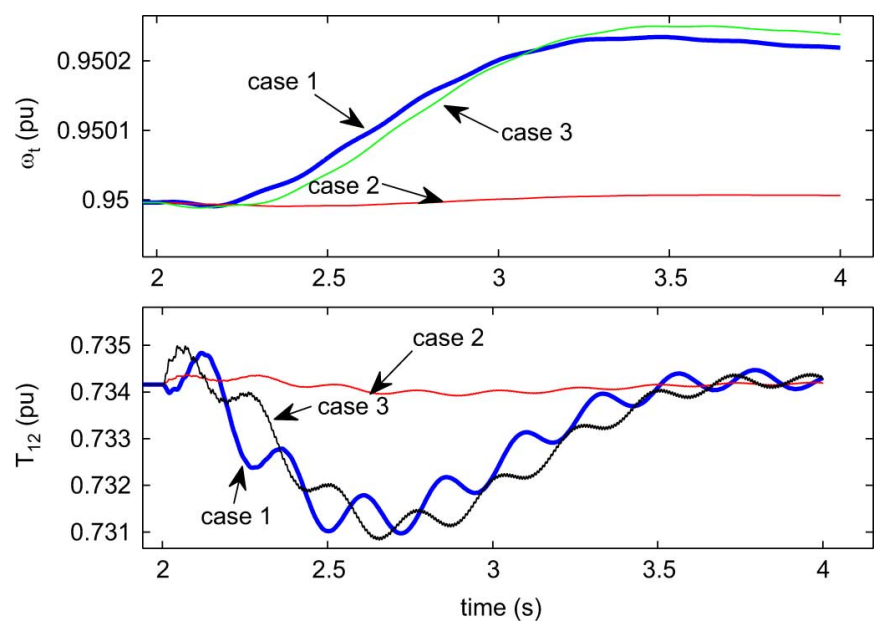

Fig. 14. Torsional speed and intermediate torque.

long as the unbalanced disturbance is presented. In addition, a $4.5-\mathrm{Hz}$ oscillation is also obvious. The SSR oscillation distorts the $120-\mathrm{Hz}$ oscillation as well. At 2.5 seconds, the distortion is no longer obvious, which indicates the die-out of the SSR mode.

Fig. 14 presents the torsional speed $\left(\omega_{t}\right)$ and the intermediate torque $T_{12}$ in the two-mass turbine. A much lower oscillation mode $(<1 \mathrm{~Hz})$ is now presented. This mode corresponds to the mechanical mode indicated in the Bode plots. The $4.5 \mathrm{~Hz}$ oscillation can be observed obviously. The comparison of three cases indicate that a 0.5 pu unbalanced disturbance has similar effect of 0.1 pu balanced disturbance. Regarding the effect on damping of these two modes, unbalanced or balanced disturbance does not have any noticed difference.

As a comparison, another scenario when the series compensation level is $50 \%$ is studied and the simulation results are presented in Figs. 15 and 16. Observing Case 1 in Fig. 15 can find that the SSR mode is now with a frequency of $27 \mathrm{~Hz}$. This observation corroborates with the analysis by Bode plots in Section III that when series compensation level increases, the 


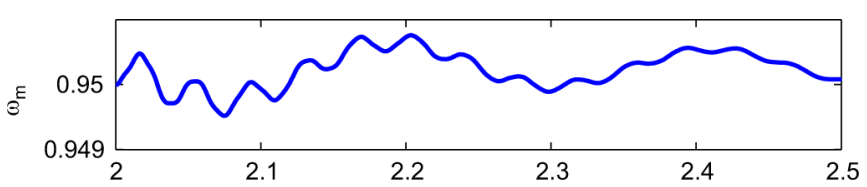

(a)

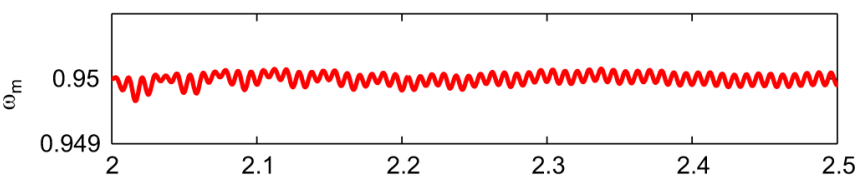

(b)

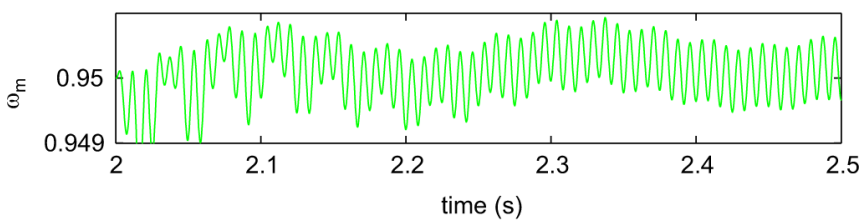

(c)

Fig. 15. Rotating speed when subject to a system voltage disturbance. Compensation level 50\%. (a) Case 1. (b) Case 2. (c) Case 3.
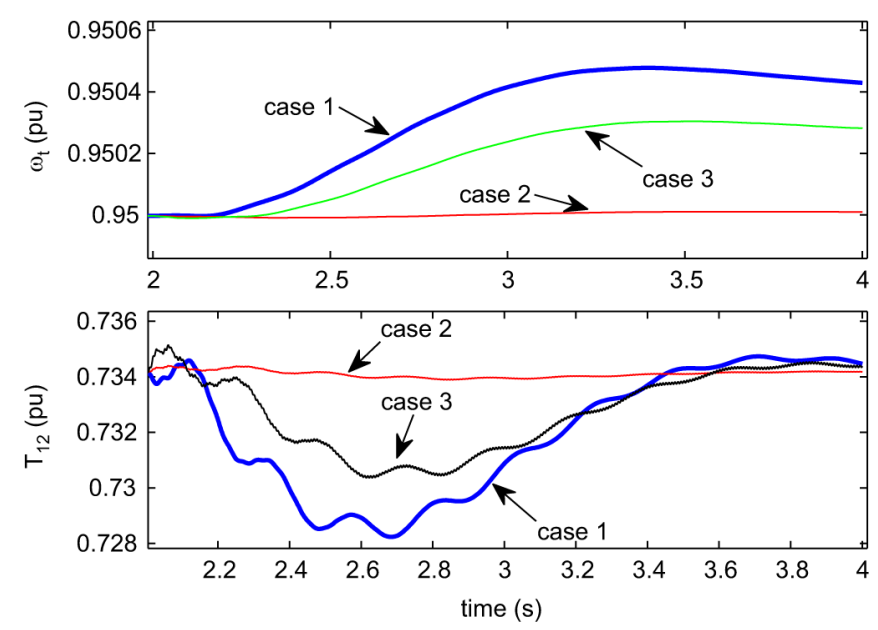

Fig. 16. Torsional speed and intermediate torque. Compensation level $50 \%$.

SSR mode frequency decreases in torque. Comparison of the dynamic response of $T_{12}$ in Fig. 16 indicates that the $0.5-\mathrm{Hz}$ and $5-\mathrm{Hz}$ oscillations are all presented in balanced and unbalanced cases.

Fig. 17 present the simulation results of electric power and electromagnetic torque. In both cases, $36-\mathrm{Hz}$ SSR mode can be observed in the balanced case and unbalanced case. Though unbalanced case has sustained $120-\mathrm{Hz}$ oscillation.

The above simulation results demonstrate that except an addition of $120-\mathrm{Hz}$ oscillation, unbalanced disturbance does not worsen the other oscillation modes. It is also understandable that the simulation case is a small disturbance case, therefore the system characteristic will be determined by the operating point which is balanced operation.

Comparison cases also indicate that a 5 times larger unbalanced disturbance could have comparable impact as a balanced disturbance on the system if the $120 \mathrm{~Hz}$ oscillation is excluded.
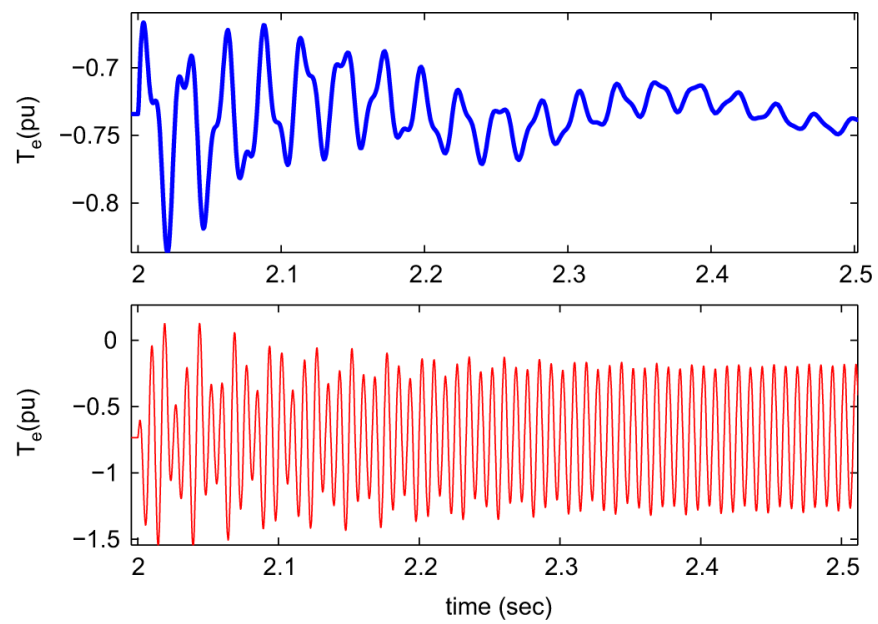

Fig. 17. Dynamic response of electromagnetic torque of DFIG. Compensation level 25\%. (a) Case 1. (b) Case 3.

TABLE I

PARAMETERS OF A SINGLE 2 MW DFIG AND THE AGGREGATED DFIG IN NETWORK SYSTEM

\begin{tabular}{|c|c|c|}
\hline Rated power & $2 \mathrm{MW}$ & $100 \mathrm{MW}$ \\
\hline Rated voltage & $690 \mathrm{~V}$ & $690 \mathrm{~V}$ \\
\hline$X_{l s}$ & $0.09231 \mathrm{pu}$ & $0.09231 \mathrm{pu}$ \\
\hline$X_{M}$ & $3.95279 \mathrm{pu}$ & $3.95279 \mathrm{pu}$ \\
\hline$X_{l r}$ & $0.09955 \mathrm{pu}$ & $0.09955 \mathrm{pu}$ \\
\hline$R_{s}$ & $0.00488 \mathrm{pu}$ & $0.00488 \mathrm{pu}$ \\
\hline$R_{r}^{\prime}$ & $0.00549 \mathrm{pu}$ & $0.00549 \mathrm{pu}$ \\
\hline$H$ & $3.5 \mathrm{~s}$ & $3.5 \mathrm{~s}$ \\
\hline$X_{t g}$ & $0.3 \mathrm{pu}(0.189 \mathrm{mH})$ & $0.3 \mathrm{pu}\left(\frac{0.189}{5} \mathrm{mH}\right)$ \\
\hline \multicolumn{3}{|c|}{} \\
\hline DC link capacitor C & $14000 \mu F$ & $50 \times 14000 \mu F$ \\
\hline DC link rated voltage & $1200 \mathrm{~V}$ & $1200 \mathrm{~V}$ \\
\hline \hline
\end{tabular}

TABLE II

PARAMETERS OF THE SHAFT SYSTEM

\begin{tabular}{|c|c|}
\hline$H_{1}$ & $0.9 \mathrm{~s}$ \\
\hline$H_{2}$ & $4.29 \mathrm{~s}$ \\
\hline$D_{1}$ & $0 \mathrm{pu}$ \\
\hline$D_{2}$ & $0 \mathrm{pu}$ \\
\hline$D_{12}$ & $1.5 \mathrm{pu}$ \\
\hline$K_{12}$ & $0.15 \mathrm{pu}$ \\
\hline
\end{tabular}

\section{CONCLUSION}

The stability impact of unbalanced operation on Type 3 wind energy systems is investigated in this paper. Two aspects are examined: the impact on electrical resonances using impedance model and the impact on torsional resonances using linear torque/speed transfer function. Negative sequence impedance models are developed and Nyquist stability criterion is applied to detect electrical resonance. Electromagnetic torque/speed transfer function is developed for negative sequence components' contribution and the effect on torque/speed is also identified. The finding of this paper indicates that negative sequence components due to unbalanced operation have insignificant impact on both electrical and torsional resonances. The analysis is further verified by time-domain simulation results. 
TABLE III

Parameters of THE NeTWORK System

\begin{tabular}{|c|c|}
\hline Transformer ratio & $690 \mathrm{~V} / 161 \mathrm{kV}$ \\
\hline Base MVA & $100 \mathrm{MVA}$ \\
\hline$R_{L}$ & $0.02 \mathrm{pu}(5.1842 \Omega)$ \\
\hline$X_{L}$ & $0.5 \mathrm{pu}(129.605 \Omega)$ \\
\hline$X_{C}$ at $50 \%$ compensation level & $64.8 \Omega$ \\
\hline Series compensation C & $40 \mu \mathrm{F}$ \\
\hline Line length & $154 \mathrm{mile}$ \\
\hline
\end{tabular}

\section{APPENDIX}

Table I lists the parameters of a single 2 MW DFIG and the aggregated DFIG in network system; Table II lists the parameters of the shaft system; Table III lists the parameters of the network system.

\section{REFERENCES}

[1] G. Irwin, A. Jindal, and A. Isaacs, "Sub-synchronous control interactions between type 3 wind turbines and series compensated ac transmission systems," in Proc. IEEE Power \& Energy Soc. General Meeting 2011, Jul. 2011.

[2] L. Fan, R. Kavasseri, Z. Miao, and C. Zhu, "Modeling of DFIG-based wind farms for SSR analysis," IEEE Trans. Power Del., vol. 25, no. 4, pp. 2073-2082, Oct. 2010.

[3] L. Fan, C. Zhu, Z. Miao, and M. Hu, "Modal analysis of a DFIG-based wind farm interfaced with a series compensated network," IEEE Trans. Energy Convers., vol. 26, no. 4, pp. 1010-1020, Dec. 2011.

[4] L. Fan and Z. Miao, "Nyquist criterion based explanation for type 3 wind generator SSR problem," IEEE Trans. Energy Convers., accepted for publication.

[5] Z. Miao, "Impedance model based SSR analysis for type 3 wind generator and series compensated network," IEEE Trans. Energy Convers., submitted for publication.
[6] L. Harnefors, "Modeling of three-phase dynamic systems using complex transfer functions and transfer matrices," IEEE Trans. Ind. Electron., vol. 54, no. 4, pp. 2239-2248, Aug. 2007.

[7] L. Harnefors, M. Bongiorno, and S. Lundberg, "Input-admittance calculation and shaping for controlled voltage-source converters," IEEE Trans. Ind. Electron., vol. 54, no. 6, pp. 3323-3334, Dec. 2007.

[8] M. Cespedes and J. Sun, "Modeling and mitigation of harmonic resonance between wind turbines and the GRI," Proc. IEEE Energy Conversion Congr. Expo. (ECCE), Sep. 2011.

[9] P. Krause, Analysis of Electric Machinery. New York, NY, USA: McGraw-Hill, 1986.

[10] A. Tabesh and R. Iravani, "Frequency-response analysis of torsional dynamics," IEEE Trans. Power Syst., vol. 19, no. 3, pp. 1430-1437, Aug. 2004.

[11] W. Qiao, W. Zhou, J. A. adn, and R. G. Harley, "Wind speed estimation based sensorless output maximization control for a wind turbine driving a DFIG," IEEE Trans. Power Electron., vol. 23, no. 3, pp. 1156-1169, May 2008.

[12] P. Kundur, Power System Stability and Control. New York, NY, USA: McGraw-Hill, 1994.

[13] L. Harnefors, "Analysis of subsynchronous torsional interaction with power electronic converters," IEEE Trans. Power Syst., vol. 22, no. 1, pp. 305-313, Feb. 2007.

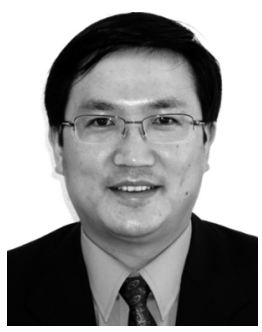

Zhixin Miao (S'00-M'03-SM'09) received the B.S.E.E. degree from the Huazhong University of Science and Technology, Wuhan, China, in 1992, the M.S.E.E. degree from the Graduate School, Nanjing Automation Research Institute, Nanjing, China, in 1997, and the Ph.D. degree in electrical engineering from West Virginia University, Morgantown, WV, USA, in 2002

Currently, he is with the University of South Florida (USF), Tampa, FL, USA. Prior to joining USF in 2009, he was with the Transmission Asset Management Department with Midwest ISO, St. Paul, MN, USA, from 2002 to 2009 . His research interests include power system stability, microgrid, and renewable energy. 